\title{
Evaluation of the effect of the administration of a glucosamine-containing supplement on biomarkers for cartilage metabolism in soccer players: A randomized double-blind placebo-controlled study
}

\author{
AKIFUMI TSURUTA ${ }^{1}$, TAKUMI HORIIKE $^{2}$, MASAFUMI YOSHIMURA $^{2}$ and ISAO NAGAOKA ${ }^{1}$ \\ ${ }^{1}$ Department of Host Defense and Biochemical Research, Juntendo University Graduate School of Medicine, \\ Tokyo 113-8421; ${ }^{2}$ Juntendo University Graduate School of Health and Sports Science, Chiba 270-1695, Japan
}

Received April 2, 2018; Accepted July 25, 2018

DOI: $10.3892 / \mathrm{mmr} .2018 .9396$

\begin{abstract}
A randomized double-blind placebo-controlled clinical study was conducted to evaluate the chondroprotective action of glucosamine on healthy subjects (soccer players) without joint disorders. Collegiate soccer players $(n=43)$ without joint disorders were randomly assigned to receive a glucosamine ( $2 \mathrm{~g}$ /day)-containing supplement $(\mathrm{n}=22$, glucosamine group) or a placebo ( $\mathrm{n}=21$, placebo group) for 16 weeks, and cartilage metabolism was evaluated by analyzing markers for type II collagen degradation urine C-terminal telopeptide-II (CTX-II) and serum collagen type II cleavage (C2C) and synthesis urine $\mathrm{C}$-terminal type II procollagen peptide (CPII). In the initial analysis of all subjects, urine CTX-II level substantially decreased in the glucosamine group, but not in the placebo group after the intervention for 16 weeks $(\mathrm{P}=0.05)$. Moreover, CTX-II level in the glucosamine group was also significantly lower than that in the placebo group at week 16 during the intervention. In the second analysis, to make the effect of the test supplement more clear, 41 subjects with less variation of exercise loading were evaluated. The results revealed that urine CTX-II level significantly decreased in the glucosamine group $(n=21)$, but not in the placebo group $(n=20)$ after the intervention $(\mathrm{P}<0.05)$. Moreover, CTX-II levels in the glucosamine group significantly decreased compared with the placebo group after the intervention $(\mathrm{P}<0.05)$. Both in the initial and second analyses, serum $\mathrm{C} 2 \mathrm{C}$ level significantly decreased in the glucosamine group, but not in the placebo group after the intervention $(\mathrm{P}<0.05)$. In contrast, urine CPII level was not significantly changed even after the intervention in both the placebo and glucosamine groups. Importantly, no
\end{abstract}

Correspondence to: Professor Isao Nagaoka, Department of Host Defense and Biochemical Research, Juntendo University Graduate School of Medicine, 2-1-1 Hongo, Bunkyo, Tokyo 113-8421, Japan E-mail: nagaokai@juntendo.ac.jp

Key words: glucosamine, cartilage metabolism, biomarkers, joint health, athlete, randomized controlled study test supplement-related adverse events were observed. These observations suggest that oral administration of glucosamine ( $2 \mathrm{~g} /$ day for 16 weeks) exerts a chondroprotective action on healthy subjects (soccer players) without joint disorders. This effect was achieved by improving cartilage metabolism (suppressing type II collagen degradation but maintaining type II collagen synthesis), without causing apparent adverse effects.

\section{Introduction}

The severity and frequency of joint loading are principal factors for the development of joint destruction, which is characterized by the articular cartilage damage. Actually, excessive motion and load on the joint cause the articular cartilage damage (1-4). Thus, sports with repetitive impact and torsional loading on the joints enhance the risk of articular cartilage degeneration, and result in the clinical symptoms of osteoarthritis (4). The pathological process of osteoarthritis leads to the degradation and functional loss of joint cartilage. Notably, in the experimental osteoarthritis models the early changes of the cartilage metabolism can be detected before the appearance of morphological changes of cartilage (2).

Thus, a number of biomarkers with reliability and sensitivity have been developed as indicators of cartilage and bone metabolism in subjects with joint and bone disorders (5). In this context, sports-related mechanical loading on the joints is shown to modulate the metabolism (turnover) of matrices of bone as well as cartilage in humans, and these changes can be detected by using biomarkers (1-4). Namely, type II collagen is a major component of cartilage matrix (6), and fragments of type II collagen have been used as biomarkers for cartilage metabolism (breakdown and synthesis of cartilage matrix). A C-terminal telopeptide (CTX-II) is cleaved during breakdown of type II collagen (7), whereas a neoepitope (C2C) is cleaved from the $\mathrm{C}$ terminus of the $3 / 4$ piece of degraded type II collagen (8). Thus, both CTX-II and C2C are utilized as markers for type II collagen degradation. In contrast, a C-terminal type II procollagen peptide (CPII) is localized in newly formed type II collagen and cleaved during processing of synthesized type II procollagen; thus, CPII can be utilized 
as a marker for type II collagen synthesis (9). Additionally, a cross-linked N-terminal telopeptides of type I collagen (NTx) can be utilized as a marker for type I collagen degradation in bone (bone resorption) (5).

Nutritional supplements containing such as glucosamine, chondroitin and collagen are often administered for "joint health' of sports-related cartilage injuries (osteoarthritis) in athletes (10-13). Among these substances, glucosamine, an amino monosaccharide, has been widely used to prevent or treat osteoarthritis in humans (14-17). Glucosamine is a component of glycosaminoglycans in the connective and cartilage tissues, and contributes to maintaining the elasticity, flexibility and strength of these tissues. Several clinical studies revealed the significant symptom-relieving and structure-modifying effects of glucosamine in osteoarthritis (14-17). Interestingly, glucosamine suppresses the degradation and augments the synthesis of glycosaminoglycans (proteoglycans) in vitro $(18,19)$. In addition, glucosamine suppresses the expression of collagen-degrading enzymes (matrix metalloproteinases) but enhances the expression of type II collagen in chondrocytes in vitro $(20,21)$. Based on these findings, glucosamine is expected to exert a chondroprotective action by maintaining proteoglycans as well as type II collagen in the articular cartilage.

Among sports with various different frequency and intensity of joint loading, soccer is categorized as a representative with high levels of repetitive impact and torsional loading on the joint (4). Thus, to investigate the effect of glucosamine on articular cartilage in endurance athletes, we previously measured the levels of biomarkers for type II collagen degradation (CTX-II) and synthesis (CPII) in an open-label (unblinded) study using soccer players (22). The results indicated that glucosamine administration $(1.5 \mathrm{~g}$ and $3 \mathrm{~g} /$ day for 3 months) significantly decreased the CTX-II level without affecting the CPII level, suggesting that glucosamine exerts a chondroprotective action in endurance athletes (soccer players) by preventing type II collagen degradation but maintaining type II collagen synthesis. However, the effect of glucosamine has not yet been confirmed in a randomized controlled trial. Thus, in the present study, we performed a randomized double-blind placebo-controlled trial, and determined the levels of type II collagen degradation (CTX-II and C2C) and synthesis (CPII) markers before and after the administration of a placebo or glucosamine ( $2 \mathrm{~g} /$ day)-containing supplement for 4 months (16 weeks) to soccer players.

\section{Materials and methods}

Study design. A prospective randomized double-blind placebo-controlled, parallel-group comparative study was designed to assess the actions of a glucosamine-containing supplement and a placebo diet on the cartilage metabolism (type II collagen degradation and synthesis) in healthy soccer players without symptoms of joint disorders. Additionally, the safety of the test supplement was evaluated. The study was registered at the UMIN Clinical Trials Registry (trial no. UMIN000023852) on August 31, 2016, and performed from August 2016 to February 2017 at Juntendo University, Japan. The study protocol with the title of 'Evaluation of a jelly-type functional food on the bone and cartilage metabolism in athletes' (protocol number: 20160703) was approved on August 10, 2016 by the Ethics Committee of The Japan Society of Vascular Medicine and Rheology (Tokyo, Japan), and the study was conducted in accordance with the principles of the amended Declaration of Helsinki and 'Ethical Guidelines for Epidemiological Research' (established by the Japanese Government in 2008). Written informed consent was obtained from all participants prior to their enrollment in the study. The whole design of the study consisted of a 3-week run-in (screening) period, a 16-week (4-month) intervention period, and a 8-week follow-up period without intervention. Subjects were screened at a baseline visit by a symptom questionnaire and routine laboratory tests. Additionally, laboratory tests were performed at weeks $0,4,8,12$ and 16 during the intervention, and 4 and 8 weeks after the intervention for the subjects.

Subjects. Selection criteria contained the following: i) Players belonging to the soccer team of Juntendo University School of Health and Sports Science; ii) nutritionally healthy adults with 18 years of age or older and iii) individuals who can participate in the study, comprising of 8 times of urine collection, 2 times of blood sampling and intake of test supplement for 4 months, with the test period of about 7 months.

Exclusion criteria contained the following: i) Current diagnosis and medication of bone or cartilage disorders including arthritis, fracture and distortion; ii) history of surgical treatment of gastric disorders, or current diagnosis of gastric disorders; iii) routine use of dietary supplements containing glucosamine or any other constituents of the test supplement, which likely influence cartilage and bone metabolism; iv) medication likely to influence cartilage and bone metabolism; v) hypersensitivity or allergy to the test components; vi) diagnosis or current medication of disorders including malignancies, hypertension (atherosclerosis), cardiac, renal, thyroid, lung and hepatic disorders; vii) pregnant women, nursing mothers or women intending to have children during the study periods; viii) blood donation $>400 \mathrm{ml}$ within 4 months prior to the study; ix) participation in any other clinical studies within one month prior to enrollment; and x) the presence of any clinical conditions judged by the medical investigators to preclude the participation of subjects in the study.

Following the assessment of 45 subjects for eligibility, one subject declined to participate of his own volition. Finally, 44 male players [aged 18-22 years; mean age, 20.1 \pm 1.1 years (mean $\pm \mathrm{SD})$ ] were enrolled as eligible subjects. All subjects were actively training for soccer during the study period: They performed the training session four times a week (from Tuesday to Friday) for approximately $2 \mathrm{~h} /$ day and played the official match every Saturday or Sunday. The research co-coordinators randomly assigned the eligible subjects to receive a placebo (22 subjects in the placebo group) or glucosamine-containing supplement (22 subjects in the glucosamine group; Fig. 1) using a table of random numbers. The allocation table was sealed, and all research staffs (which included medical investigators, clinical service staffs, analyzers of urine and blood samples, and other research staffs) and participants were blinded to the allocation during 


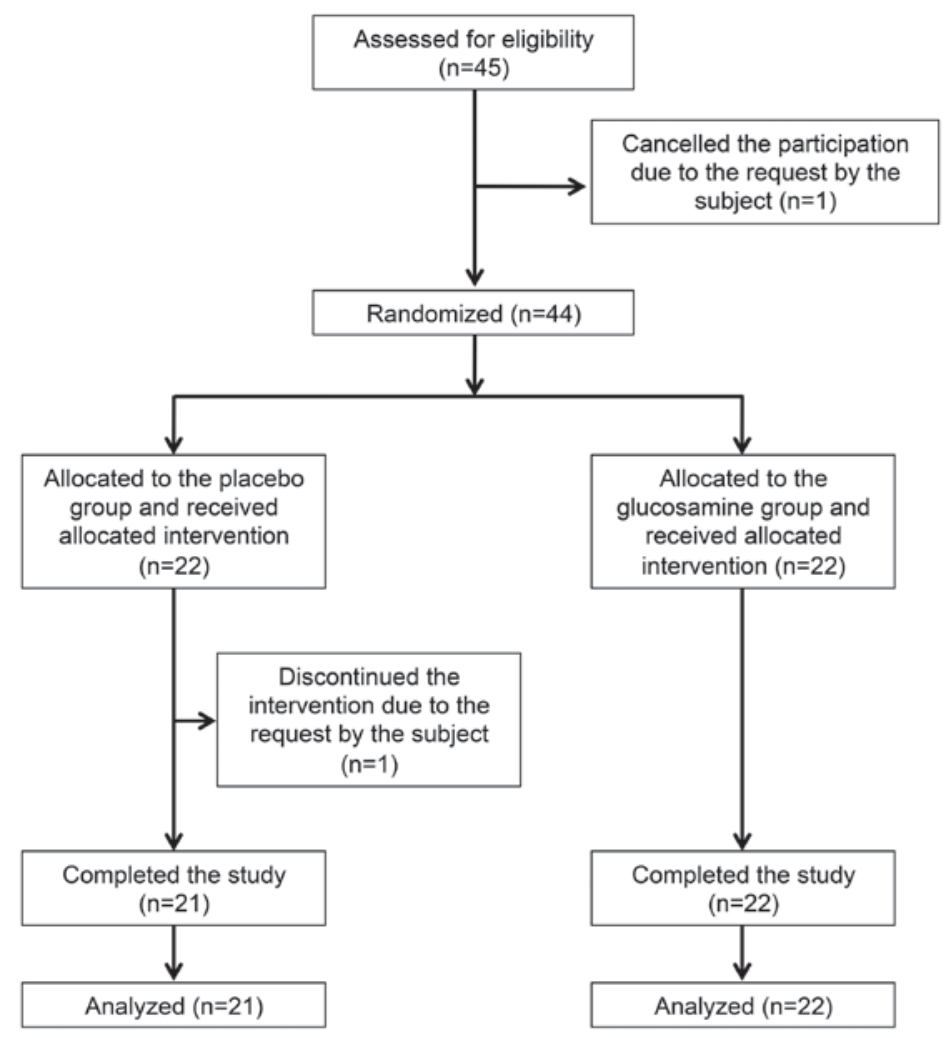

Figure 1. Flow diagram of the subjects, who participated in the study.

the test period. Following completion of the study, the allocation table was made available for analysis of the data. During the intervention, one subject discontinued the study of his own volition in a placebo group. Thus, 43 subjects completed the study (mean age $20.1 \pm 1.1$ years; 21 subjects in the placebo group and 22 subjects in the glucosamine group), and were finally judged to be eligible for assessment of the efficacy of test supplement (Fig. 1 and Table I). Moreover, to improve the clarity regarding the effect of test supplement, the subjects with less variation of exercise loading, based on the changes of type II and type I collagen degradation markers (CTX-II and NTx, respectively) (5), were analyzed. Thus, 2 subjects (one subject in a placebo group and one subject in a glucosamine group) with augmented exercise loading during the test period, as evidenced by the increased levels of both CTX-II and NTx (>1.8-fold), were excluded, and 41 subjects with less variation of exercise loading (mean age, 20.2 \pm 1.1 years; 20 subjects in the placebo group, 21 subjects in the glucosamine group) were evaluated (Table II).

Intervention and subject assignment. The participants were instructed not to alter their lifestyles, including habits of eating, sleeping and drinking alcohol, and especially exercise training. They were also instructed not to start taking any dietary supplements during the test period, or not to stop taking dietary supplements if they already started taking dietary supplements. The test supplement was manufactured in a jelly type (180 $\mathrm{g}$ in an aluminum pouch with a spout) by Toppan Packaging Service Co., Ltd., (Tokyo, Japan) and contained 2,000 mg glucosamine hydrochloride, $1,800 \mathrm{mg}$ arginine, $600 \mathrm{mg}$ lysine hydrochloride, $400 \mathrm{mg}$ alanine,
$400 \mathrm{mg}$ glycine, $10 \mathrm{mg}$ hesperidin, $0.2 \mathrm{mg} \beta$-cryptoxanthin and a vehicle consisting of $14 \mathrm{~g}$ maltodextrin, $4 \mathrm{~g}$ fructose, $4 \mathrm{~g}$ glucose and $5 \mathrm{~g}$ sucrose, whereas the placebo diet $(180 \mathrm{~g})$ contained only a vehicle consisting of $14 \mathrm{~g}$ maltodextrin, $5 \mathrm{~g}$ fructose, $4 \mathrm{~g}$ glucose and $11 \mathrm{~g}$ sucrose.

Subjects were randomly assigned to receive a 2,000 $\mathrm{mg}$ glucosamine-containing supplement (glucosamine group) or a placebo containing only a vehicle (placebo group). All subjects were instructed to take the test supplement or placebo once a day within $30 \mathrm{~min}$ after exercise for 16 weeks. The daily dose of glucosamine (2,000 mg/day) was determined based on the results of the previous study (22). Adherence to the intervention was evaluated on the basis of consumption record in the study diary.

Second void of morning urine was collected after an overnight fast at a baseline visit, weeks $0,4,8,12$, and 16 during the intervention, and 4 and 8 weeks after the intervention. Serum was collected from the subjects in a fasting state at weeks 0 and 16 during the intervention. Serum and urine samples were immediately used for routine laboratory tests; alternatively, serum and urine samples were aliquoted and stored at $-80^{\circ} \mathrm{C}$ until the assays of CTX-II, C2C and CPII.

Evaluation of efficacy and safety. To evaluate the effect of test supplement on the cartilage metabolism, urine and serum samples collected at weeks 0 and 16 during the intervention were used for the assays of type II collagen degradation (urine CTX-II and serum C2C) and synthesis (urine CPII) markers. Urine CTX-II was measured using a Urine Cartilaps (CTX-II) EIA kit (Immunodiagnostic Systems Limited, Boldon, UK), which detects a C-terminal telopeptide (CTX-II) of type II 
Table I. Baseline characteristics of the subjects in the placebo and glucosamine groups.

\begin{tabular}{lccc}
\hline Characteristics & Placebo $(\mathrm{n}=21)$ & Glucosamine $(\mathrm{n}=22)$ & P-value \\
\hline Ages (years) & $20.1 \pm 1.2$ & $20.2 \pm 1.0$ & 0.48 \\
Height $(\mathrm{cm})$ & $173.0 \pm 5.5$ & $174.9 \pm 4.9$ & 0.70 \\
Weight $(\mathrm{kg})$ & $67.1 \pm 6.0$ & $68.9 \pm 5.1$ & 0.56 \\
BMI $\left(\mathrm{kg} / \mathrm{cm}^{2}\right)$ & $22.4 \pm 1.1$ & $22.5 \pm 1.1$ & 0.92 \\
NTX $(\mathrm{nmol} \mathrm{BCE} / \mathrm{mmol} \mathrm{Cr})$ & $99.0 \pm 27.7$ & $77.0 \pm 26.6$ & 0.85 \\
CTX-II $(\mathrm{ng} / \mathrm{mmol} \mathrm{Cr})$ & $1460.9 \pm 1036.5$ & $1248.6 \pm 657.9$ & 0.04 \\
CPII $(\mathrm{ng} / \mathrm{mmol} \mathrm{Cr})$ & $3294.2 \pm 1866.7$ & $2837.2 \pm 1990.3$ & 0.78 \\
C2C $(\mathrm{ng} / \mathrm{ml})$ & $22.3 \pm 7.0$ & $20.9 \pm 9.5$ & 0.18 \\
\hline
\end{tabular}

Values are expressed as the mean \pm standard deviation. Baseline characteristics of subjects were analyzed by Student's t-test between the placebo and glucosamine groups. BMI, body mass index; NTx, type I collagen degradation marker; CTX-II and C2C, type II collagen degradation markers; CPII, type II collagen synthesis marker.

Table II. Baseline characteristics of the subjects in the placebo and glucosamine groups, with less variation in exercise loading during the test period.

\begin{tabular}{lccr}
\hline Characteristics & Placebo $(\mathrm{n}=20)$ & Glucosamine $(\mathrm{n}=21)$ & P-value \\
\hline Ages (years) & $20.2 \pm 1.2$ & $20.2 \pm 1.0$ & 0.45 \\
Height $(\mathrm{cm})$ & $173.0 \pm 5.5$ & $175.1 \pm 5.1$ & 0.70 \\
Weight $(\mathrm{kg})$ & $67.1 \pm 6.0$ & $69.0 \pm 5.3$ & 0.54 \\
BMI $\left(\mathrm{kg} / \mathrm{cm}^{2}\right)$ & $22.4 \pm 1.1$ & $22.5 \pm 1.2$ & 0.85 \\
NTX $(\mathrm{nmol} \mathrm{BCE} / \mathrm{mmol} \mathrm{Cr})$ & $101.9 \pm 25.0$ & $77.9 \pm 26.9$ & 0.76 \\
CTX-II $(\mathrm{ng} / \mathrm{mmol} \mathrm{Cr})$ & $1510.1 \pm 1038.0$ & $1250.7 \pm 674.1$ & 0.06 \\
CPII $(\mathrm{ng} / \mathrm{mmol} \mathrm{Cr})$ & $3356.6 \pm 1892.6$ & $2826.8 \pm 2038.8$ & 0.75 \\
C2C $(\mathrm{ng} / \mathrm{ml})$ & $22.3 \pm 7.2$ & $20.7 \pm 9.7$ & 0.20 \\
\hline
\end{tabular}

Values are expressed as the mean \pm standard deviation. Baseline characteristics of subjects were analyzed by Student's t-test between the placebo and glucosamine groups. BMI, body mass index; NTx, type I collagen degradation marker; CTX-II and C2C, type II collagen degradation markers; CPII, type II collagen synthesis marker; BCE, bone collagen equivalent; $\mathrm{Cr}$, creatinine.

collagen (7). Serum C2C was measured using a Collagen Type II Cleavege ELISA kit (IBEX Pharmaceuticals Inc., Montreal, Canada), which detects a neoepitope created by the collagenase cleavage of type II collagen (8). Urine CPII was measured using a Procollagen type II C-propeptide ELISA kit (IBEX Pharmaceuticals), which detects a C-terminal propeptide of newly formed type II procollagen (C-propeptide, also referred as CPII) (9).

CTX-II, C2C and CPII were measured in duplicates on the same microtiter plate. Concentrations of urine CTX-II and CPII were corrected by urinary creatinine $(\mathrm{Cr})$, and expressed as ng/mmol $\mathrm{Cr}$.

Cross-linked N-terminal telopeptides of type I collagen (NTx) is excreted in urine during bone degradation (degradation of type I collagen), and can be used as markers of bone turnover (resorption) (5). Urinary NTx and creatinine were measured by LSI Medience Corporation (Tokyo, Japan), based on the ELISA (NTx) and enzyme assay (creatinine), respectively, and the concentrations of NTx was expressed as nmol $\mathrm{BCE}$ (bone collagen equivalent)/mmol $\mathrm{Cr}$, after correction with urinary creatinine.
Safety and tolerability were assessed throughout the study, based on the incidence and severity of intervention-related adverse events (side effects), as well as abnormal changes in laboratory tests, including hematology, biochemical profile and urinalysis. Change in the physical conditions and use of pharmaceutical products were also recorded in a diary by the participants.

Statistical analysis. Values are expressed as the means \pm standard deviation (SD). In the baseline characteristics of subjects, parameters were analyzed by Student's t-test (Prism 5; GraphPad Software, San Diego, CA, USA) between the placebo and glucosamine groups. The levels of CTX-II, C2C, CPII and NTx were compared between weeks 0 and 16 in the placebo or glucosamine group, and between the placebo and glucosamine groups at weeks 0 and 16 during the intervention by two-way analysis of variance with Tukey's post hoc test. Additionally, safety data were compared between the placebo and glucosamine groups by Student's t-test. $\mathrm{P}<0.05$ was considered to indicate a statistically significant difference. 


\section{Results}

Characterization of study groups. Table I shows the baseline characteristics of 43 subjects (21 subjects in the placebo group; 22 subjects in the glucosamine group), who completed the study and fulfilled the eligibility criteria. The baseline characteristics included age, physiological characteristics (body height, body weight and body mass index) and levels of biomarkers for type II collagen metabolism (CTX-II, C2C and CPII). There were no significant differences in these parameters between the placebo and glucosamine groups at the baseline, except for CTX-II level, which was significantly lower in the glucosamine group compared with the placebo group $(\mathrm{P}=0.04)$. Adherence to the allotted dietary supplement (a placebo or a glucosamine-containing supplement) was $100 \%$ among the 43 subjects who completed the study.

Assessment of cartilage metabolism using type II collagen degradation and synthesis markers. Next, we examined the effect of test supplement on cartilage metabolism using type II collagen degradation (CTX-II and C2C) and synthesis (CPII) markers. Interestingly, urine CTX-II level substantially decreased in the glucosamine group but not in the placebo group after the intervention for 16 weeks ( $\mathrm{P}=0.05$; Fig. 2A). Moreover, CTX-II level was significantly lower in the glucosamine group than that in the placebo group at week 16 during the intervention $(\mathrm{P}<0.05$; Fig. 2A). Similarly, serum $\mathrm{C} 2 \mathrm{C}$ level significantly decreased in the glucosamine group but not in the placebo group after the intervention for 16 weeks $(\mathrm{P}<0.05)$, although C2C levels were not significantly different between the placebo and glucosamine groups at week 0 or 16 during the intervention (Fig. 2B). In contrast, urine CPII levels were not significantly different between weeks 0 and 16 during the intervention in the placebo or glucosamine group, and between the placebo and glucosamine groups at week 0 or 16 during the intervention (Fig. 2C).

Furthermore, we examined the effect of test supplement on bone metabolism using type I collagen degradation marker (NTx). Urine NTx levels were not significantly different between weeks 0 and 16 during the intervention in the placebo or glucosamine group, and between the placebo and glucosamine groups at week 0 or 16 during the intervention (Fig. 2D).

Assessment of cartilage metabolism in subjects with less variation of exercise loading. It has been reported that the level of CTX-II (type II collagen degradation in cartilage) significantly correlates with that of NTx (type I collagen degradation in bone) in endurance athletes, and the levels of CTX-II and NTx reflect the exercise loading $(3,13,23)$. Thus, in order to make the effect of the test supplement more clear, 2 subjects (one subject in the placebo group and one subject in the glucosamine group) were excluded, because both CTX-II and NTx levels (exercise loading) were markedly increased ( $>1.8$-fold) in these subjects during the intervention, and these changes may affect the efficacy of test supplement. Thus, the rest of 41 subjects with less variation of exercise loading, based on the changes of type II (CTX-II) and type I collagen (NTx) degradation markers, were evaluated (mean age, 20.2 \pm 1.1 years; 20 subjects in the placebo group, 21 subjects in the glucosamine group; Table II).
Table II presents the baseline characteristics of these subjects, including age, physiological characteristics (body height, body weight and body mass index) and levels of biomarkers for type II collagen metabolism (CTX-II, C2C and CPII). There were no significant differences in these parameters between the placebo and glucosamine groups at the baseline. Thus, the effect of test supplement on cartilage metabolism was evaluated using these subjects at weeks 0 and 16 during the intervention.

The results indicated that urine CTX-II level significantly decreased in the glucosamine group but not in the placebo group after the intervention for 16 weeks $(\mathrm{P}<0.01)$; moreover, CTX-II level was significantly lower in the glucosamine group than that in the placebo group at week 16 during the intervention $(\mathrm{P}<0.05$; Fig. 3A). Similarly, serum $\mathrm{C} 2 \mathrm{C}$ level significantly decreased in the glucosamine group but not in the placebo group after the intervention for 16 weeks $(\mathrm{P}<0.05)$, although $\mathrm{C} 2 \mathrm{C}$ levels were not significantly different between the placebo and glucosamine groups at week 0 or 16 during the intervention (Fig. 3B). In contrast, urine CPII levels were not significantly different between weeks 0 and 16 during the intervention in the placebo or glucosamine group, and between the placebo and glucosamine groups at week 0 or 16 during the intervention (Fig. 3C). Similarly, urine NTx levels were not significantly different between weeks 0 and 16 during the intervention in the placebo or glucosamine group, and between the placebo and glucosamine groups at week 0 or 16 during the intervention (Fig. 3D).

Assessment of safety and tolerability. Among 44 enrolled subjects, all subjects in the placebo group $(n=22)$ and the glucosamine group $(n=22)$ experienced essentially no adverse events during and after the intervention period. Furthermore, the physical measurement parameters (body weight and body mass index) and laboratory tests (urinalysis, hematology and blood chemistry) did not show any significant changes from the baseline during and after the intervention in the two groups.

\section{Discussion}

Glucosamine is now used as a functional dietary supplement to relieve the symptoms of osteoarthritis, based on its significant symptom-modifying action on osteoarthritis revealed by the clinical trials (14-17). Notably, the in vitro studies have indicated that glucosamine likely exhibits chondroprotective action by inhibiting the degradation and stimulating the synthesis of proteoglycans in the cartialge $(18,19)$.

Biomarkers can be used to evaluate the pathophysiological conditions of joint disorders (5). Markers are basically derived form the constituents of cartilage, such as aggrecan, chondroitin sulfate and collagens $(5,6)$. Among these constituents, type II collagen is a major constituent of articular cartilage, and the catabolism and anabolism of articular type II collagen are essentially involved in joint disorders; thus the components of type II collagen are recognized as the most important biomarkers for joint disorders (such as osteoarthritis) (24).

In an open-study, we previously evaluated the effect of glucosamine administration ( $1.5 \mathrm{~g}$ and $3 \mathrm{~g} / \mathrm{day}$ for 3 months) on the cartilage metabolism in soccer players by using 

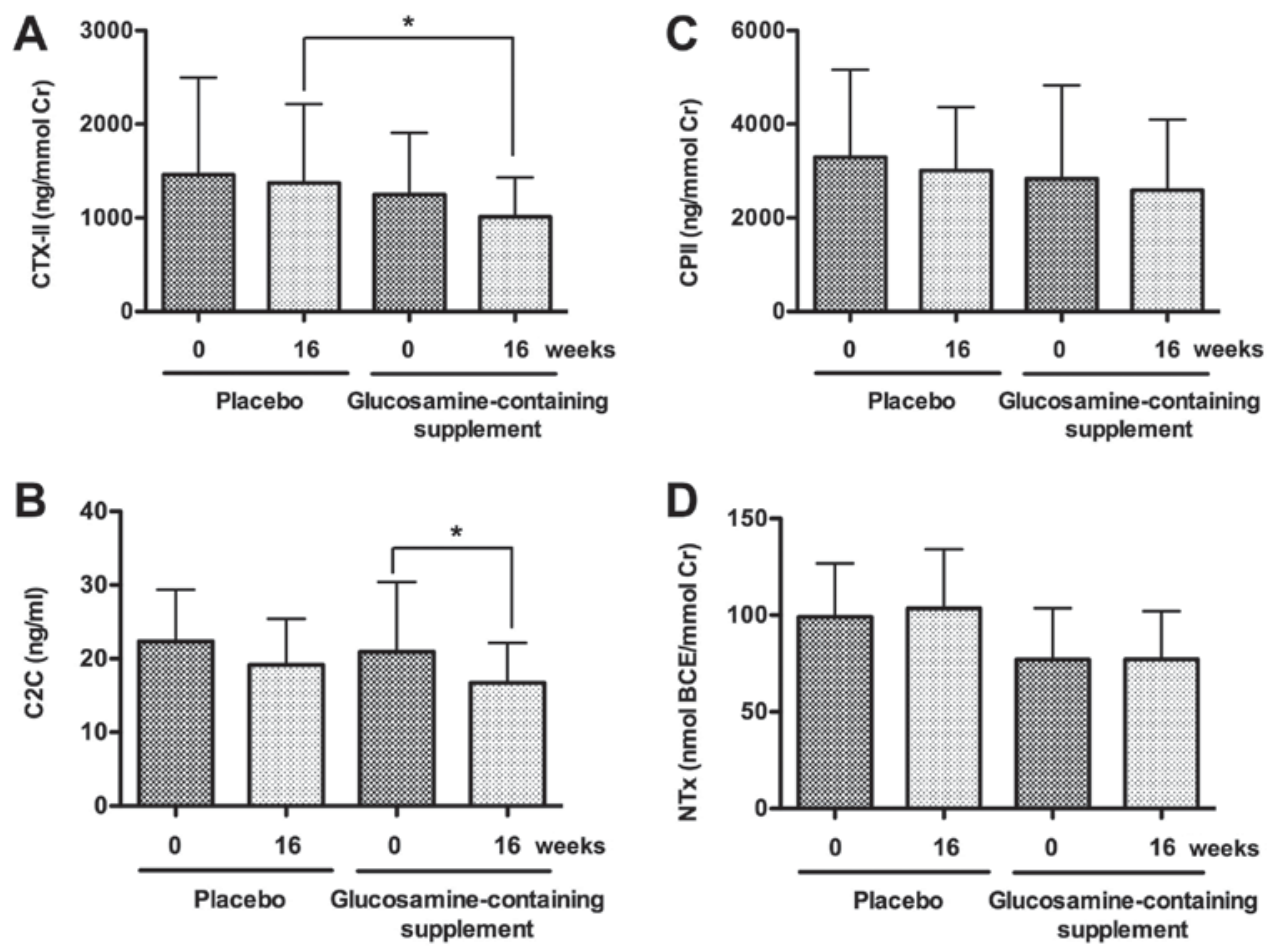

Figure 2. Effect of glucosamine administration on the biomarkers for type II collagen degradation, type II collagen synthesis and type I collagen degradation in soccer players. Soccer players were orally administered with a placebo or glucosamine-containing supplement (2 g/day for 4 months). Urine and serum samples were collected at weeks 0 and 16 during the administration from the subjects shown in Table I. Urine CTX-II (A), serum C2C (B), urine CPII (C) and urine NTx (D) were measured by ELISA. The levels of urine markers were corrected by urinary creatinine (Cr). Data are the mean \pm SD of 21 subjects in the placebo group and 22 subjects in the glucosamine group. Values were compared between weeks 0 and 16 in the placebo or glucosamine groups, and among multiple groups (such as the placebo and glucosamine groups at weeks 0 and 16 during the intervention) by two-way analysis of variance with Tukey's post hoc test. " $\mathrm{P}<0.05$.
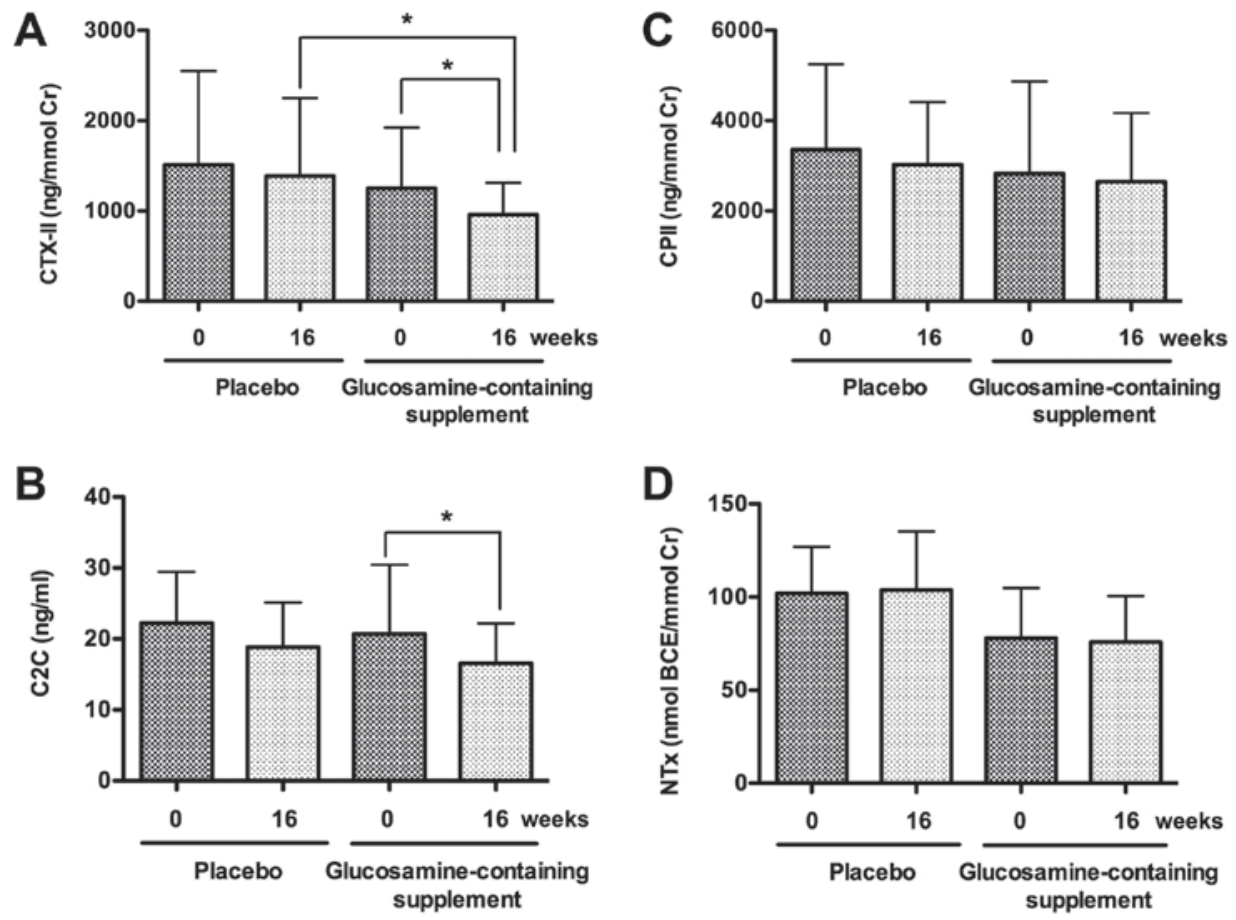

Figure 3. Effect of glucosamine administration on the biomarkers for type II collagen degradation, type II collagen synthesis and type I collagen degradation in soccer players with less variation of exercise loading. Soccer players were orally administered with a placebo or glucosamine-containing supplement ( $2 \mathrm{~g} / \mathrm{day}$ for 4 months), and urine and serum samples were collected at weeks 0 and 16 during the administration. Urine CTX-II (A), serum C2C (B), urine CPII (C) and urine NTx (D) were measured by ELISA, and the levels of urine markers were corrected by urinary creatinine (Cr). In order to make the effect of the test supplement more clear, 41 subjects with less variation of exercise loading, based on the changes of type II (CTX-II) and type I collagen (NTx) degradation markers were evaluated (as shown in Table II). Data are the mean \pm SD of 20 subjects in the placebo group and 21 subjects in the glucosamine group. Values were compared between weeks 0 and 16 in the placebo or glucosamine groups, and among multiple groups (such as the placebo and glucosamine groups at weeks 0 and 16 during the intervention) by two-way analysis of variance with Tukey's post hoc test. " $\mathrm{P}<0.05$. 
biomarkers for type II collagen-degradation (CTX-II) and -synthesis (CPII) (22), since soccer players repetitively expose their joints to impact and torsional loading and have a potential risk of articular cartilage degeneration (4). Furthermore, we evaluated the bone metabolism in soccer players by using a degradation maker (NTx) of type I collagen, a major component of bone collagen (5), since subchondral bone remodeling (accompanied with degradation of type I collagen) is developed during progression of joint disorders $(25,26)$. Moreover, we compared the basic levels of type II collagen degradation (urine CTX-II) and synthesis (urine CPII) markers and type I collagen degradation (urine NTx) marker between soccer players and non-athlete controls (22). The results indicated that the basic levels of CTX-II and NTx but not CPII are significantly higher in soccer players than in non-athlete controls, suggesting that type II collagen and type I collagen degradation is enhanced in soccer players with excessive motion and loading on the joints. Importantly, glucosamine administration (1.5 $\mathrm{g}$ and $3 \mathrm{~g} /$ day for 3 months) significantly reduced the level of CTX-II but not CPII and NTx, suggesting that glucosamine exerts a chondroprotective action on athletes (soccer players) by mainly suppressing type II collagen degradation. However, the effect of glucosamine has not yet been confirmed in a randomized controlled trial. Thus, in the present study, we performed a randomized double-blind placebo-controlled trial, and measured the biomarker levels of type II collagen degradation (CTX-II and C2C) and synthesis (CPII), and type I collagen degradation (NTx) in soccer players, before and after the administration of a placebo or glucosamine ( $2 \mathrm{~g}$ /day)-containing supplement for 16 weeks.

In the initial analysis of all the subject, who completed the study and fulfilled the eligibility criteria (Table I), urine CTX-II level substantially decreased in the glucosamine group but not in the placebo group after the intervention for 16 weeks ( $\mathrm{P}=0.05$; Fig. 2A). Unexpectedly, CTX-II level in the glucosamine group was also significantly lower than that in the placebo group at week 16 during the intervention $(\mathrm{P}<0.05$; Fig. 2A). Likewise, serum C2C level significantly decreased in the glucosamine group but not in the placebo group after the intervention for 16 weeks $(\mathrm{P}<0.05$; Fig. $2 \mathrm{~B})$. In contrast, urine CPII level as well ad urine NTx level was not significantly changed after the intervention in both the placebo or glucosamine groups (Fig. 2C and D).

In the second analysis, in order to make the effect of the test supplement more clear, 41 subjects with less variation of exercise loading, based on the changes of type II (CTX-II) and type I collagen (NTx) degradation markers, were evaluated (Table II). The results revealed that urine CTX-II level significantly decreased in the glucosamine group but not in the placebo group after the intervention for 16 weeks $(\mathrm{P}<0.05)$ (Fig. 3A). Furthermore, CTX-II level in the glucosamine group significantly decreased compared with the placebo group after the intervention for 16 weeks $(\mathrm{P}<0.05$; Fig. 3A). Also, serum $\mathrm{C} 2 \mathrm{C}$ level significantly decreased in the glucosamine group but not in the placebo group after the intervention for 16 weeks $(\mathrm{P}<0.05$; Fig. 3B). In contrast, the levels of urine CPII as well as urine NTx were not significantly changed even after the intervention in both the placebo and glucosamine groups (Fig. 3C and D). Thus, the present randomized controlled study revealed that glucosamine administration $(2 \mathrm{~g} / \mathrm{day}$ for 16 weeks) significantly reduces the levels of CTX-II and C2C but not CPII and NTx, confirming that glucosamine exerts a chondroprotective action on athletes (soccer players) by suppressing type II collagen degradation (as assessed by CTX-II and C2C, type II collagen degradation markers).

In this context, it has been reported that glucosamine suppresses the production of matrix metalloproteinase (MMP)-13, a major type II collagen-degradaing enzyme, from chondrocytes and synoviocytes in vitro $(20,21)$ and reduces the serum level of MMP-3 in sera of patients with rheumatoid arthritis (27). Based on these findings, it is interesting to speculate that glucosamine inhibits MMP production, thereby suppressing type II collagen degradation (as evidenced by the reduction of CTX-II and C2C levels) in vivo. In contrast, glucosamine administration did not basically affect the levels of CPII as well as NTx, suggesting no effect of glucosamine administration on the levels of type II collagen synthesis (CPII) and type I collagen degradation (NTx) in soccer players. Importantly, glucosamine has been reported to enhance the expression of type II collagen in chondrocytes in vitro (21); however, in the present study, the increase of type II collagen synthesis (as evaluated by CPII) could bot detected in soccer players (Figs. 1C and 2C). This is probably due to the fact that type II collagen synthesis (as evaluated by CPII) was slightly increased in soccer players than in non-athlete controls $(22,23)$, although the increase is not significant; thus, the CPII level can not be further enhanced by glucosamine administration.

In summary, this is the first randomized double-blind placebo-controlled study to demonstrate the potential effect of oral administration of glucosamine administration on the cartilage metabolism in healthy individuals (soccer players). The efficacy and safety of glucosamine administration suggest that a dietary supplement containing glucosamine ( $2 \mathrm{~g} /$ day for 16 weeks) can be safely administered and potently exerts a chondroprotective action on healthy individuals (soccer players) without symptoms of joint disorders by improving the type II collagen metabolism (suppressing type II collagen degradation but not affecting type II collagen synthesis) in the cartilage, without any adverse effects. Thus, glucosamine-containing supplement, as a functional food (28), can be considered a potential candidate for maintaining or caring the joint health of healthy individuals without joint disorders.

\section{Acknowledgements}

The authors would like to thank Mr. Motoki Kanayama, a manager of the soccer team of Juntendo University School of Health and Sports Science for helping the authors collect the urine samples and performing this randomized controlled study.

\section{Funding}

No funding was received.

\section{Availability of data and materials}

The datasets used and/or analyzed during the present study are available from the corresponding author on reasonable request. 


\section{Authors' contributions}

AT and IN designed the research; AT, TH and MY performed the clinical study; AT and IN analyzed the data; AT and IN prepared the manuscript. All authors read and approved the final manuscript.

\section{Ethics approval and consent to participate}

The study was registered at the UMIN Clinical Trials Registry (trial no. UMIN000023852) on August 31, 2016, and performed from August 2016 to February 2017 at Juntendo University, Japan. The study protocol with the title of 'Evaluation of a jelly-type functional food on the bone and cartilage metabolism in athletes' (protocol no: 20160703) was approved on August 10, 2016 by the Ethics Committee of The Japan Society of Vascular Medicine and Rheology (Tokyo, Japan), and the study was conducted in accordance with the principles of the amended Declaration of Helsinki and 'Ethical Guidelines for Epidemiological Research' (established by the Japanese Government in 2008). Written informed consent was obtained from all participants prior to their enrollment in the study.

\section{Patient consent for publication}

All the participants enrolled in the present study provided written informed consent for the publication of any associated data.

\section{Competing interests}

The authors declare that they have no competing interests.

\section{References}

1. Roos H, Dahlberg L, Hoerrner LA, Lark MW, Thonar EJ, Shinmei M, Lindqvist U and Lohmander LS: Markers of cartilage matrix metabolism in human joint fluid and serum: The effect of exercise. Osteoarthritis Cartilage 3: 7-14, 1995.

2. Qi C and Changlin H: Effects of moving training on histology and biomarkers levels of articular cartilage. J Surg Res 135: 352-363, 2006.

3. O'Kane JW, Hutchinson E, Atley LM and Eyre DR: Sport-related differences in biomarkers of bone resorption and cartilage degradation in endurance athletes. Osteoarthritis Cartilage 14: 71-76, 2006.

4. Buckwalter JA and Lane NE: Athletics and osteoarthritis. Am J Sports Med 25: 873-881, 1997.

5. Rousseau JC and Delmas PD: Biological markers in osteoarthritis. Nat Clin Pract Rheumatol 3: 346-356, 2007.

6. Poole AR: Biochemical/immunochemical biomarkers of osteoarthritis: Utility for prediction of incident or progressive osteoarthritis. Rheum Dis Clin North Am 29: 803-818, 2003.

7. Christgau S, Garnero P, Fledelius C, Moniz C, Ensig M, Gineyts E, Rosenquist C and Qvist P: Collagen type II C-telopeptide fragments as an index of cartilage degradation. Bone 29: 209-215, 2001.

8. Poole AR, Ionescu M, Fitzcharles MA and Billinghurst RC: The assessment of cartilage degradation in vivo: Development of an immunoassay for the measurement in body fluids of type II collagen cleaved by collagenases. J Immunol Methods 294: 145-153, 2004.

9. Shinmei M, Ito K, Matsuyama S, Yoshihara Y and Matsuzawa K: Joint fluid carboxy-terminal type II procollagen peptide as a marker of cartilage collagen biosynthesis. Osteoarthritis Cartilage 1: 121-128, 1993.
10. Schwenk TL and Costley CD: When food becomes a drug: Nonanabolic nutritional supplement use in athletes. Am J Sports Med 30: 907-916, 2002.

11. Gorsline RT and Kaeding CC: The use of NSAIDs and nutritional supplements in athletes with osteoarthritis: Prevalence, benefits, and consequences. Clin Sports Med 24: 71-82, 2005.

12. Ostojic SM, Arsic M, Prodanovic S, Vukovic J and Zlatanovic M: Glucosamine administration in athletes: Effects on recovery of acute knee injury. Res Sports Med 15: 113-124, 2007.

13. Nagaoka I: Joint health of athletes and the chondroprotective action of glucosamine. Juntendo Med J 63: 104-114, 2017.

14. McAlindon TE, LaValley MP, Gulin JP and Felson DT: Glucosamine and chondroitin for treatment of osteoarthritis: A systematic quality assessment and meta-analysis. JAMA 283: $1469-1475,2000$.

15. Reginster JY, Deroisy R, Rovati LC, Lee RL, Lejeune E, Bruyere O, Giacovelli G, Henrotin Y, Dacre JE and Gossett C: Long-term effects of glucosamine sulphate on osteoarthritis progression: A randomized, placebo-controlled clinical trial. Lancet 357: 251-256, 2001.

16. Pavelká K, Gatterová J, Olejarová M, Machacek S, Giacovelli G and Rovati LC: Glucosamine sulfate use and delay of progression of knee osteoarthritis: A 3-year, randomized, placebo-controlled, double-blind study. Arch Intern Med 162: 2113-2123, 2002.

17. Nagaoka I: Recent aspect of the chondroprotective and anti-inflammatory actions of glucosamine, a functional food. Juntendo Med J 60: 580-587, 2014.

18. Fenton JI, Chlebek-Brown KA, Peters TL, Caron JP and Orth MW: Glucosamine $\mathrm{HCl}$ reduces equine articular cartilage degradation in explant culture. Osteoarthritis Cartilage 8: $258-265,2000$

19. Gouze JN, Bordji K, Gulberti S, Terlain B, Netter P, Magdalou J, Fournel-Gigleux $\mathrm{S}$ and Ouzzine $\mathrm{M}$ : Interleukin-1beta down-regulates the expression of glucuronosyltransferase I, a key enzyme priming glycosaminoglycan biosynthesis: Influence of glucosamine on interleukin-lbeta-mediated effects in rat chondrocytes. Arthritis Rheum 44: 351-360, 2001.

20. Nakamura H, Shibakawa A, Tanaka M, Kato T and Nishioka K: Effects of glucosamine hydrochloride on the production of prostaglandin E2, nitric oxide and metalloproteases by chondrocytes and synoviocytes in osteoarthritis. Clin Exp Rheumatol 22: 293-299, 2004.

21. Derfoul A, Miyoshi AD, Freeman DE and Tuan RS: Glucosamine promotes chondrogenic phenotype in both chondrocytes and mesenchymal stem cells and inhibits MMP-13 expression and matrix degradation. Osteoarthritis Cartilage 15: 646-655, 2007.

22. Yoshimura M, Sakamoto K, Tsuruta A, Yamamoto T, Ishida K, Yamaguchi $\mathrm{H}$ and Nagaoka I: Evaluation of the effect of glucosamine administration on biomarkers for cartilage and bone metabolism in soccer players. Int J Mol Med 24: 487-494, 2009.

23. Nagaoka I, Tsuruta A and Yoshimura M: Evaluation of cartilage and bone metabolism in collegiate athletes belonging to various sports clubs by analyzing type II collagen degradation and synthesis and type I collagen degradation. Juntendo Med J 64 (Suppl 1): 122-127, 2018.

24. Garnero P, Piperno M, Gineyts E, Christgau S, Delmas PD and Vignon E: Cross sectional evaluation of biochemical markers of bone, cartilage, and synovial tissue metabolism in patients with knee osteoarthritis: Relations with disease activity and joint damage. Ann Rheum Dis 60: 619-626, 2001.

25. Radin EL and Rose RM: Role of subchondral bone in the initiation and progression of cartilage damage. Clin Orthop Relat Res: 34-40, 1986.

26. Bettica P, Cline G, Hart DJ, Meyer J and Spector TD: Evidence for increased bone resorption in patients with progressive knee osteoarthritis: Longitudinal results from the Chingford study. Arthritis Rheum 46: 3178-3184, 2002.

27. Nakamura H, Masuko K, Yudoh K, Kato T, Kamada T and Kawahara T: Effects of glucosamine administration on patients with rheumatoid arthritis. Rheumatol Int 27: 213-218, 2007.

28. Martirosyan DM and Singh J: A new definition of functional food by FFC: What makes a new definition unique? Functi Foods Health Dis 5: 209-223, 2015. 